\title{
Desarrollo histórico de la primera ley de la termodinámica y algunas implicaciones didácticas
}

\author{
Historical Development to the first law of the Thermodynamic and some didactic \\ implications
}

Jenny Alexandra Muñoz Pérez

Universidad Pedagógica Nacional. jamp qui@hotmail.com

\section{Resumen}

En el presente ensayo se realiza una breve descripción histórica relacionada con el desarrollo de la primera ley de la termodinámica y su debate entre los principales investigadores de la época, en relación con los conceptos de calor, energía libre y entalpía. Además sus implicaciones didácticas con relación a los errores conceptuales que los estudiantes presentan al momento de abordar esta temática.

\section{Palabras clave}

Termodinámica, calor, historia, energía libre, implicación didáctica.

\section{Abstract}

In this essay is a brief description with the historical development of the first law of thermodynamics and the debate between the principal investigators of the time, in connection with the heat, free energy and enthalpy. Besides its educational implications in relation to conceptual errors that students have time to address this issue.

\section{Keywords}

Thermodynamics, heat, history, free energy, involving didactic.

\section{Introducción}

La introducción del primer principio de la Termodinámica y/o de los principales conceptos implicados (como el trabajo, el calor, la energía interna y la entalpía) en la enseñanza convencional de la Química se hace sin tener en cuenta la historia de las ciencias y los resultados de la investigación didáctica sobre los nuevos modelos de aprendizaje de orientación constructivista. Eso hace que no se favorezcan en los estudiantes de bachillerato y universitarios la comprensión de los intercambios energéticos en los procesos fisicoquímicos (Furió, Solves, 1997).

Este ensayo, tratará de darificar cómo se han construido los conocimientos termodinámicos enfocados a la primera ley de al termodinámica, desde la teoría del calórico, pasando por la energía interna, hasta la entalpía. Además se incluyen algunos problemas de aprendizaje en la parte conceptual y epistemológica que surgen en la enseñanza - aprendizaje de la termodinámica. 
Cuestión de la teoría del calórico, su principal contribución al desarrollo de la termodinámica

Con los conocimientos actuales de la estructura moleaular de la materia, se dice que el flujo de calor entre dos cuerpos implica una transferencia de energía intema entre ellos. Sin embargo, en los siglos XVIII y XIX, la teoría molecular de la materia era objeto de gran controversia. La termodinámica nace, a principio del siglo $\mathrm{XIX}$, como una gran síntesis que trató de unificar la explicación de las diferentes fuerzas introducidas en los procesos mecánicos, eléctricos, químicos, térmicos y magnéticos. Esta síntesis se suele comparar con la hecha por la mecánica newtoniana entre la dinámica celeste y terrestre y comenzó por el proceso de unificación de los estudios del calor y de la mecánica considerados como ciencias separadas (Arons, 1970). De este apartado podemos identificar algunos problemas conceptuales que se generan para la diferenciación de las nociones de calor y temperatura que presentan los estudiantes que se enfrentan por primera vez a la temática de termodinámica.

El primer problema que se generó a partir de esta controversia, fue la diferenciación entre el concepto de calor y la temperatura. Gracias a la construcción y uso de termómetros, Joseph Black (1728-1799) definió operacionalmente la temperatura de un cuerpo como el número que marcaba el termómetro puesto contacto con él y el calor se entendía como algo que se intercambiaban los auerpos a diferente temperatura hasta llegar al equilibrio térmico. Con esta experimentación, relacionaron el concepto de calor con la variación de temperatura y la masa del cuerpo calentado o enfriado. Se fue construyendo la calorimetría como un método (de mezclas) que permitía predecir y contrastar la temperatura de equilibrio al poner en contacto dos cuerpos a diferente temperatura.

En relación a la naturaleza del calor aunque, Bacon, Hooke y Newton mantenían en el siglo XVII que "el calor era una propiedad del auerpo calentado resultando del movimiento (vibratorio) 0 agitación de sus partes" (Arons, 1970), durante el siglo XVIII habían proliferado muchas teońas, tanto en electricidad como en química y en calor, basadas en fluidos. Así, por ejemplo, los efectos eléctricos eran descritos mediante el comportamiento de los fluidos y efluvios que intercambiaban los cuerpos electrizados y lo mismo ocurría en la explicación de los efectos magnéticos (Furió y Guisasola, 1998).

La naturaleza del calor se conoció hasta aproximadamente 1850, esto comenzó con la combustión de la materia orgánica que era explicada por los químicos de la época donde explicaban el intercambio de una especie de fluido denominado 'flogisto' y concebido como el principio de inflamabilidad de los cuerpos. En el caso del calor, se postulaba la "teoría del calórico" en la que se suponía que el calor era una sustancia material formada por partículas que se repelían entre sí pero que eran atraídas por las partículas de las sustancias ordinarias. La mayor o menor atracción entre las partículas de calórico y de las sustancias explicaba la diferente capacidad calorífica de éstas; también se reconocía, el calórico como una sustancia fluida hipotética presente en la materia que supuestamente fluía de un cuerpo caliente a uno frío. La cantidad de calórico perdida por el cuerpo caliente era igual a la ganada por el frío. Se creía que la cantidad total de calónico se conservaba en todos los procesos (Levine, 2000). Este modelo también daba cuenta de la distinción entre "calor sensible" y "calor latente" en función del tipo de unión de los átomos de calórico con los de los materiales. En el caso del calor sensible, los átomos ordinarios se rodeaban de una atmósfera de calórico y en el del calor latente, se unían de manera más fuerte semejante a las combinaciones químicas (Arons, 1970).

Así fue como la teoría del flogisto fue puesta seriamente en cuestión por Lavoisier con sus investigaciones sobre la combustión y sobre la oxidación de los metales. Uno de los pocos investigadores que puso en cuestión el carácter material del calórico al final del siglo XVIII fue el norteamericano Benjamin Thompson (1753-1814), más conocido como conde de Rumford. El estaba interesado en el estudio de sistemas de calentamiento a vapor, construcción de chimeneas, 
etc. y es sabido que, haciendo de supervisor de la perforación de cañones de bronce en los talleres del arsenal militar de Munich (Baviera), puso en auestión el carácter sustancial del calórico al considerar que la fuente de calor generado por la frotación del aparato que cortaba las virutas en el bronce de los cañones era inagotable, la principal hipótesis era considerar que el calor fuera movimiento. A finales del siglo XVIII, la mayoría de los cient́ficos aceptaban la teoría del calórico.

\section{Significado de energía interna, calor y temperatura.}

La caítica del conde de Rumford sobre la naturaleza sustancial del calórico fue continuada ya en el siglo XIX, donde se buscaban explícitamente relaciones entre la mecánica, el calor, la electricidad y la química. Por el mismo siglo, Julius Mayer, observó que la comida que consumimos, se emplea en gran parte para mantener la temperatura del arerpo, $y$ en parte para producir el trabajo mecánico realizado por el organismo. Con esta experiencia que se vive diariamente, propuso entonces que el trabajo y el calor eran dos formas de energía y que la cantidad total de energía se conservaba. Los argumentos planteados por Mayer no fueron tan convincentes y fue James Joule quien tuvo que continuar con el problema del calórico.

Joule llevó a cabo una serie de experimentos y demostró que los mismos cambios tienen lugar al calentar una sustancia, también se puede producir al realizar un trabajo mecánico sobre ella sin transferencia de calor. Su experimento más conocido era el del uso de pesos que bajaban para mover una ruedas dentro de líquidos. La energía potencial de los pesos se convertía en energía cinética del líquido, y la fricción interna de este transformaba su energía cinética en energía intema, produciendo un aumento de temperatura (Levine, 2000).

La energía interna de un sistema puede variar de diferentes maneras; puesto de que se trata de una propiedad extensiva, depende de la cantidad de materia del sistema. Además de la posibilidad de variar las masas del sistema mediante la adición o extracción de materia, se puede cambiar la energía del mismo mediante la realización de trabajo sobre él o calentándolo. La primera ley de la termodinámica afirma que existe una función de estado extensiva $E$ (llamada energía total del sistema) tal que para un sistema cerrado:

$$
\Delta E=q+\mathbf{w}
$$

Donde $\Delta E$ es el cambio de energía del sistema en el proceso, $q$ es el calor transferido al sistema durante el proceso y $w$ es el trabajo realizado sobre e sistema en el transcurso del proceso. Si se genera en un sistema en reposo, cenrado y sin campos, tenemos que $E=U$, generándose la equación

$$
\Delta \mathbf{U}=\mathbf{q}+\mathbf{w}
$$

La energía interna $U$ es una función del estado del sistema. En cambio los valores de q y w no son funciones de estado, ya que no tiene sentido preguntar auanto calor o cuanto trabajo contiene un sistema.

A pesar de que se dice a menudo que calor y trabajo son formas de energía, puede inducir al error de considerar el calor y el trabajo como funciones de estado. El calor y el trabajo están definidos solo en términos de procesos; antes y después del proceso de transferencia de energía entre el sistema y su entomo, el calor y el trabajo no existen. El calor es una transferencia de energía entre el sistema y el medio debido a su diferencia de temperatura. El trabajo es una transferencia de energía entre el sistema y sus alrededores debida a una fuerza macroscópica que actúa a lo largo de una distancia (Levine, 2000). Cuando cuerpos de diferente temperatura se ponen e contacto, las colisiones entre las moléculas de los dos cuerpos provocan una transferencia de energía del auerpo más caliente al más frío. El calor es el trabajo realizado a nivel molecular. 
Con esto, a principios de los años 1840 auando Joule y Mayer establecen una relación cuantitativa de equivalencia entre el trabajo y el calor, y comienza a introducirse el concepto de energía diferenciándolo de fuerza y a abandonarse el modelo del calórico.

Es interesante recordar que la principal obra de Mayer, Comentarios sobre las fuerzas (energías) de naturaleza inorgánica apareció en Annalen der Chemie und Pharmacie y tenía como objetivo explicar porque el calor espeáfico a volumen constante de los gases era menor que el calor específico a presión constante. Esta explicación consistía en aceptar que en la expansión térmica de un gas a presión constante, el calor suministrado en exceso se convertía en trabajo hecho por el gas al expansionarse contra la presión atmosférica (Arons, 1970). Basándose en esta relación Mayer obtuvo un valor del equivalente mecánico del calor que resultó muy semejante al obtenido posteriormente por Joule por diferentes métodos eléctricos y mecánicos ya conocidos.

Es en esta década de 1840 auando la ciencia deriva de la relación entre trabajo y calor, surgió el concepto de energía como una función general de los sistemas que les permite hacer trabajo. Definición limitada, de entrada, a la 'fuerza viva' (energía cinética) y a los cambios mecánicos y que, irá evolucionando hasta llegar a definirla como la capacidad de los sistemas para hacer transformaciones en particular, haciendo trabajo y/o transfiriendo calor- según el primer principio de la termodinámica. El concepto energía se convierte en estructurante pues sirve para explicar, en general, las diferentes interacciones que se habían ido introduciendo por las distintas ciencias (mecánica, electricidad, magnetismo y química) y cualquier tipo de cambio.

\section{Entalpía de un sistema: termodinámica de las reacciones químicas}

A continuación se establece una aproximación a la idea de la entalpía de la época, para la cual se han desarrollado diversos eventos que se presentan a continuación (Furió, Solves, 2007):

* La idea del fuego continuaba siendo interpretada por la teoría del "flogisto" de Stahl (1697), como "materia del fuego" por su disápulo Boerhaave (1732) e, incluso, como sustancia simple, el "calórico", en el Tratado elemental de Química de Antoine Laurent Lavoisier (1743-1794).

* Respecto a los intercambios energéticos en los procesos químicos, tanto Lavoisier como Laplace propusieron que el calor producido en una reacción química estaba asociado a las fuerzas de la afinidad de los reaccionantes coherente con el marco de la mecánica newtoniana.

* Hess asume la hipótesis de Lavoisier y estudia más detenidamente el calor de reacción cuando mide el calor de dilución de disoluciones acuosas de ácido sulfúrico de diferentes concentraciones añadiéndoles partes alícuotas de agua pura. Este investigador suponía que las disoluciones eran 'compuestos imperfectos' de acuerdo con la hipótesis de Claude Louis Berthellot (1748-1822) en la controversia con Proust a finales del XVIII y constató que la cantidad de calor producido en la dilución era constante e independiente del camino (por ejemplo, en sucesivas diluciones), puesto que sólo dependía de los estados inicial y final del proceso.

* Más adelante, Julius Thomson explicitó la idea de que el calor liberado era realmente una medida de la afinidad química, enunciando además el que después será denominado por Berthellot como principio del máximo trabajo:

"Para que una reacción química se pueda producir a una temperatura mantenida invariable, es necesario que esta reacción venga acompañada de un desprendimiento de calor". 
El mismo Duhem critica esta afirmación y menciona que este principio debe restringirse a procesos químicos de gran 'vivacidad' y solo puede utilizarse por los químicos, en estos casos, para ver el sentido de la reacción posible. Presenta, al mismo tiempo, un conjunto de compuestos aryas reacciones de formación son endotérmicas como, por ejemplo, el anhídrido hipodoroso, el ozono y el agua oxigenada que también se producen aunque son inestables.

$>$ En trabajos publicados entre 1862 y 1866, Berthellot afirmó que parte del calor medido por métodos termoquímicos podía venir del trabajo mecánico o eléctrico hecho sobre el sistema. El resume en tres principios sus concepciones sobre la interpretación energética de por qué ocurren las reacciones químicas:

1. El trabajo molecular o el calor obtenido en el proceso químico es una medida del trabajo físico y químico hecho en el curso de la reacción. Y es una medida de la afinidad química.

2. El calor absorbido o liberado en un cambio químico (aislado) depende exdusivamente del estado inicial y final del sistema y no de los estados intermedios.

3. El principio de máximo trabajo: Cada cambio químico realizado sin la acción de energía extema tiende a producir un cuerpo o un conjunto de cuerpos con el máximo calor posible: Cada reacción química que puede hacerse sin trabajo inicial y sin la acción de energía externa sobre los auerpos presentes en el sistema, puede darse espontáneamente si da calor al exterior.

Como se ve, Berthellot define el calor que se desarrolla en una reacción química como una función de estado en el segundo principio y, de otra parte, también se puede observar que utiliza el calor y el trabajo indistintamente, tal vez por influencia de Joule. En efecto, el primer principio de Berthellot es una derivación de la equivalencia entre el calor desprendido y el trabajo molecular en las reacciones. Este podríamos decir que es el primer precedente en el que se relaciona el calor obtenido en una reacción química con el trabajo de rotura de las uniones entre los átomos de las moléculas que forman las sustancias reaccionantes. Esta es otra dificultad que también se puede presentar en el aprendizaje en el que se asocia directamente la producción de calor con la rotura de enlaces moleculares, sin tener en cuenta energéticamente la nueva formación de uniones atómicas. En relación a los calores de reacción a presión constante, los termodinámicos derivaron de la primera ley de la termodinámica un nuevo potencial energético,

$$
H=U+P . V
$$

al que, ya entrado el siglo XX, el holandés Kamerling Onnes denominó entalpía de un sistema, $\boldsymbol{H}$. La variación de entalpía, $\Delta H$, que se produce al reaccionar las sustancias fue definida operacionalmente como el calor de reacción siempre que se realice el proceso a presión constantey se puede medir en un calorímetro. No obstante, la nueva función de estado puede tener dificultades en la comprensión de su significado, en particular, por el segundo sumando $P$. V. En primer lugar, hay que recordar el campo de validez de aquella definición operacional

$$
\mathbf{q p}=\Delta \mathbf{H}
$$

ya que se introduce a partir del primer principio de la termodinámica imponiendo algunas restricciones al sistema como son que en la interacción solo se pueda producir calor y trabajo de expansión-compresión. Por lo tanto, si, por ejemplo, en el proceso se hace trabajo eléctrico o se producen otras radiaciones no consideradas como calor, no será válido igualar el calor de reacción a presión constante y la variación de entalpía. Por otro lado, mientras no hay ningún problema en el significado aualitativo de la energía.

En resumen, el significado cualitativo atribuido a la entalpía de un sistema que va a interaccionar con otro sería el de un potencial energético suma de otros dos potenciales, uno derivado de su energía intema (U) y un segundo, el potencial P.V. Este último potencial supone admitir la 
posibilidad de hacer trabajo de compresión-expansión sobre el sistema por otro extemo (por ejemplo, el entorno atmosférico), siempre que la presión sea constante.

\section{Implicaciones en la enseñanza y didáctica de las ciencias}

A partir de la anterior aproximación histórica que se enfoca en el primer principio de la termodinámica, podemos determinar que uno de los principales aspectos que toca la didáctica de las ciencias, son las visiones deformadas que tenemos los profesores sobre la ciencia y como se construyen los conocimientos científicos que tienen importancia porque se suelen transmitir consciente 0 inconscientemente en la enseñanza de las ciencias, como han tratado diferentes investigadores. Se tendrán en cuenta diversas visiones que se pueden desplegar en la enseñanza de la termodinámica y como la historia de la ciencia puede contribuir a superarlas (Solbes y Traver, 1996 y 2003):

> Una visión descontextualizada socialmente de la ciencia que ha mostrado el movimiento investigador de las interacciones CTSA, sugiere la necesidad de hacer una educación científica más social que muestre los problemas del desarrollo tecnológicos y científicos y de sus consecuencias tanto positivas como negativas; es necesario despertar el interés y la motivación de los estudiantes hacia las ciencias y su aprendizaje. Con la enseñanza de la termoquímica se pueden encontrar situaciones problemáticas de interés personal y social que pueden ir desde el papel de las máquinas térmicas en la revolución industrial, la necesidad de entender su funcionamiento para mejorar su bajo rendimiento, como hicieron Sadi Carnot y otros.

> La visión empirista y ateórica del profesorado de ciencias bastante investigada en la didáctica (Matthews 1994) y donde se prioriza el denominado por Piaget 'mito sensorial en la construcción de los conocimientos científicos; es decir, sí es como nuestra mente funciona atando ideas o estructuras mentales ya establecidas en nuestro pensamiento Esa puede ser la razón por la que algunos conceptos cientúficos se ligan fácilmente con definiciones procedimentales de bajo nivel cognitivo y no lo hacen con definiciones relacionales de los mismos conceptos de mayor nivel. Por ejemplo, la definición procedimental de la temperatura como el número que marca el termómetro introducida por Bladk no tiene ninguna dificultad para los estudiantes pero, al relacionarlo con el concepto de calor ya se encuentra un poco confuso. Lo mismo pasó, como se vio en la breve fundamentación histórica, con el concepto de entalpía de un sistema que tiene bastantes dificultades de comprensión y, en cambio, resulta mucho más fácil la definición procedimental de la variación de entalpía de un proceso. En efecto, esta variación se mide con el calor de reacción a presión constante pero, al mismo tiempo, se presenta el problema conceptual de identificar enróneamente entalpía con calor.

> La visión excesivamente formalista de los conocimientos científicos como la que ocurre habitualmente en la introducción de conceptos en Termoquímica donde se abusa de un operativismo ciego que, a veces, llega a ser dogmático. Es frecuente en este dominio derivar matemáticamente un concepto sin tener previamente alguna idea o representación cualitativa de él (Toulmin, 1977) o sin presentar antes los problemas, fenómenos o referentes empíricos que se quieren explicar con aquel concepto científico. Por ejemplo, está el caso de la introducción del concepto de entalpía de un cambio sin haber mostrado previamente los fenómenos caloríficos y/o mecánicos (haciendo trabajo) que acompañan a los procesos físicos 0 químicos, interacciones que conforman el problema general que se quiere estudiar y explicar desde el punto de vista energético, como hemos mostrado en la anterior presentación histórica.

> La visión ahistórica y aproblemática en la introducción de los conceptos y teorías científicas que no muestra como, por ejemplo, el origen de un concepto es la respuesta hipotética dada por el cuerpo teórico a uno o varios problemas existentes en un momento dado. Es habitual ver como 
la enseñanza de las ciencias olvida el carácter problemático e histórico de los conceptos, es decir, que estos nacen intentando solucionar problemas, se desarrollan, cambian e, incluso, pueden llegar a desaparecer (Solbes y Traver, 1996 y 2003).

\section{Conclusiones}

Con el conocimiento del desarrollo histórico, se puede contribuir a mejorar la introducción del primer principio de la Termodinámica y de los principales conceptos implicados (como el trabajo, el calor, la energía intema y la entalpía), a la enseñanza convencional de la termoquímica, que lastimosamente se hace sin tener en cuenta dicha historia. Además del componente histórico, se debe reforzar en la parte experimental para que a partir de sus aproximaciones, creen analogías y representaciones macroscópicas y microscópicas de los diferentes sistemas que se pueden representar en un aula de dase.

\section{Bibliografía}

Aduriz Bravo, A. y Galagovsky, L. (1997). Modelos científicos y modelos didácticos en la enseñanza de las ciencias naturales. Actas X REF 1c 05.

Arons, A.B. (1970). Evolución de los conceptos de la Física. México: Ed. Trillas.

Furió, C., Calatayud, M.L., Guisasola, J. \& Furió-Gómez, C. (2005). How are the Concepts and Theories of Acid-Base Reactions Presented? Chemistry in Textbooks and Presented by Teachers. International Joumal of Science Education, 27 (11). 1337-1359.

Furió-Gómez, C. Solbes. J. Furió-Mas, C. (2007). La historia del primer principio de la termodinámica y sus implicaciones didácticas. Revista Eureka. Enseñ. Divul. Cien., 4(3), pp. 461475.

Johnstone, A.H. (1993). Development of Chemistry Teaching. Joumal of Chemical Education, 70, 701-703.

Levine, I. (2000). Fisicoquímica. Cuarta edición. Volumen 1. Mc Graw Hill.

Matthews, M. R. (1994). Historia, filosofía y enseñanza de las ciencias: la aproximación actual. Enseñanza de las Ciencias, 12 (2), 255-277.

Solbes, J. y Traver, M. (1996). La utilización de la historia de las Ciencias en la enseñanza de la física y química. Enseñanza de las Ciencias, 14(1), 103-112.

Solbes, J. y Traver, M. (2003). Against a Negative Image of Science: History of Science and the Teaching of Physics and Chemisty. Science \& Education, 12 (7), 703-717.

Toulmin, C. (1977). La comprensión humana. I: el uso colectivo y la evolución de los conceptos. Madrid: Alianza Editor. 